DOI: $10.3901 / J M E .2019 .15 .154$

\title{
基于广义杆组对多环路三移一转位移 输出机构的型综合*
}

张 兴 ${ }^{1}$ 牟德君 ${ }^{2,3}$ 张一同 ${ }^{2,3}$ 李维奇 ${ }^{2,3}$ 王洪瑞 ${ }^{1}$

(1. 燕山大学工业计算机控制工程河北省重点实验室 秦皇岛 066004;

2. 燕山大学河北省并联机器人与机电系统实验室 秦皇岛 066004;

3. 燕山大学先进锻压成型技术与科学教育部实验室 秦皇岛 066004)

摘要: 基于广义杆组提出了一种对多环路三移一转位移输出机构型综合的新方法。该方法把运动链当成广义杆组逐个添加 在输出构件上, 进一步扩展了 Assar 杆组法在空间的应用。分析了单环路到多环路杆组自由度与输出构件自由度和位移参 数的关系, 得到了添加自由度为 0 的广义杆组对输出构件自由度和位移参数的变化关系。综合了三移一转位移参数的杆组, 建立了交集运算规则。通过组合两个广义杆组综合输出构件自由度为 $2 、 3 、 4$ 单环路机构, 再添加自由度为 0 广义杆组, 得到了双环路机构。通过自由度为 0 广义杆组综合出多环路机构的例证, 展现了该方法在空间机构综合方面的全面性和有 效性。

关键词: 型综合; 广义杆组; 多环路; 三移一转; 位移参数 中图分类号: TP242

\section{Type Synthesis of Multi-loop Mechanisms with Three Translational and One Rotational Displacement Parameters Based on Generalized Link Group}

ZHANG Xing $^{1}$ MU Dejun ${ }^{2,3}$ ZHANG Yitong ${ }^{2,3}$ LI Weiqi ${ }^{2,3}$ WANG Hongrui $^{1}$

(1. Key Lab of Industrial Computer Control Engineering of Hebei Province, Yanshan University,

Qinhuangdao 066004;

2. Key Lab of Parallel Robot and Mechatronic System, Yanshan University, Qinhuangdao 066004;

3. Key Lab of Advanced Forging \& Stamping Technology and Science of Ministry of National Education, Yanshan University, Qinhuangdao 066004)

\begin{abstract}
A new method to synthesize multi-loop mechanisms with three translational and one rotational displacement parameters is proposed in this paper. This method is based on generalized link group. It extends the unified link groups in plane and space by using kinematic links as a generalized link group added one-by-one to the output link. The internal relation on the change in the degree of freedom (DOF) and displacement parameters of the output link after adding a 0-DOF generalized link group are analyzed. The link groups with three translational and one rotational displacement parameters are synthesized, and its intersection operation rules are established. The 2-, 3-, and 4-DOF single-loop mechanisms are obtained by combining two generalized link groups. The dual-loop mechanisms are synthesized by adding a 0-DOF generalized link group. The multi-loop mechanisms are obtained by adding 0-DOF generalized link groups. Some novel mechanisms are synthesized to illustrate the effectiveness and comprehensiveness of the proposed method.
\end{abstract}

Key words: type synthesis; generalized link group; multi-loop; three translational and one rotational; displacement parameter

\section{0 前言}

型综合是机械设计的基础, 过去几十年，引起

* 国家自然科学基金(61473112)和河北省自然科学基金(E2011203193)资 助项目。20180828 收到初稿, 20181123 收到修改稿
了许多学者的关注, 并提出许多与之对应的自由度 公式和型综合方法, 机构型综合主要有以下三种方 法: 螺旋理论 ${ }^{[1-5]}$; 位移子群 ${ }^{[6-11]}$ 和图论 $^{[12-14]}$ 。基于 螺旋理论, 黄真等 ${ }^{[1-2]}$ 提出了约束螺旋综合理论, 系 统的综合出了 9 种类型的少自由并联机构。KONG 等 ${ }^{[3-4]}$ 基于螺旋理论提出了虚拟支链的综合方法来 
代替复杂支链去综合并联机构。方跃法等 ${ }^{[5]}$ 也利用 螺旋理论综合了对称的四自由度和五自由度机器 人; 利用李群和李代数, HERVÉ ${ }^{[6]}$ 提出了位移子群 综合法, 给出了 12 种位移子群代替不同的空间位 移。 $\mathrm{RICO}^{[7]}$ 在分析空间位移子群及位移子集的基础 上, 对不同的并联机构进行了构型综合的比较。李 泽湘等 ${ }^{[8]}$ 利用微分流形理论扩展位移子群, 并提出 了微分几何综合法。李秦川等 ${ }^{[9-11]}$ 在此基础上应用 位移子群方法综合了不同类型的空间机构; 基于图 论, EARL 等 ${ }^{[12]}$ 也综合了并联机构, 但其方法依赖 于自由度计算公式, 难以确定输出构件的运动类型。 LU 等 ${ }^{[13-14]}$ 提出系统连杆法和拓扑矩阵-图表法来综 合并联机构, 并应用该方法综合了 3、4 自由度的并 联机构。此外, GOGU 等 ${ }^{[15]}$ 通过对雅可比矩阵的线 性变换来综合机构。杨廷力等 ${ }^{[16]}$ 提出了基于方位特 征综合法对并联机构进行了综合。高峰等 ${ }^{[17-18]}$ 提出 了 $G_{f}$ 集的概念来综合并联机构。

除了以上不同的数学工具和综合方法, ASSAR $^{[19]}$ 提出了 Assar 杆组的概念来综合机构, 该 方法是在原来的开链上添加不影响机构自由度的运 动链。此后, MANOLESCU ${ }^{[20]}$ 应用了 Assar 杆组法 综合了带有 8,9 和 10 个杆的运动链。CAMPOS ${ }^{[21]}$ 把 Assar 杆组当作基本块来综合混联机构。 $\mathrm{LI}^{[22]}$ 提 出基于杆组邻接矩阵的平面机构综合的方法, 使基 于 Assur 杆组的结构组成理论的应用进一步系统化 和计算机化。然而, Assar 杆组法不适用于一般的空 间机构。而且很难得出输出构件的运动类型。其主 要问题在于自由度公式不能反映杆组自由度与输出 构件自由度和位移参数的关系。

张一同等 ${ }^{[23-26]}$ 提出了新的自由度计算方法虚 拟环路法。该公式反映了杆组自由度、输出构件自 由度和输出构件位移参数之间的关系。同时, 把空 间单开链定义为广义杆组。基于此, 本文进一步研 究了三移一转位移参数输出机构中自由度为 0 的 广义杆组与输出构件的位移参数和自由度的关系。 通过在输出构件逐个添加广义杆组来综合空间并 联机构。

少自由度并联机构中, 三移一转并联机构广泛 应用于电子、食品、包装等领域。其中许多三移一 转机构被综合出来 $\left(\mathrm{H} 4^{[27]}, \mathrm{Par} 4^{[28]}, \mathrm{I}^{[29]}, \mathrm{SCARA}^{[30]}\right.$, $4-\mathrm{UPU}^{[2]}, 4-\mathrm{RRRRR}^{[3]}$, Quadrupteron ${ }^{[31]}$ 和 Isoglide-4 ${ }^{[15]}$ 机构)。事实上, 许多综合方法应用于三移一转机

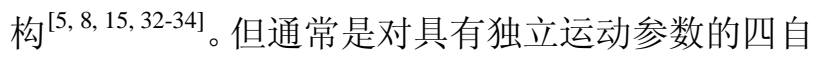
由度三移一转机构的综合。而对二、三自由度具有 非独立参数的机构研究的非常少。然而, 这类机构 能够实现三移一转的位移。由于这些机构有驱动少,
结构简单等优点, 能够应用于农业和矿山工业的振 动篎中。因此, 研究三移一转位移输出并联机构有 着重要意义。

为此, 针对多环路三移一转位移输出机构, 本 文提出了基于虚拟环路法的型综合方法。其主要内 容如下: 第 1 节介绍了广义杆组的基本理论, 建立 交集运算规则, 综合了三移一转位移参数杆组。第 2 节分析了从单环路到多环路杆组自由度与输出构 件自由度和位移参数的关系。第 3 节对自由度为 2 、 3、4 的单环路机构进行综合。第 4 节, 添加自由度 为 0 的不同类型的广义杆组, 得到双环路的机构。 第 5 节, 添加自由度为 0 的杆组得到多环路机构。 第 6 节, 得出结论。

\section{1 广义杆组}

\section{1 杆组自由度和位移参数表达}

在文献[23]中, 单开链可以看成广义杆组, 简 称杆组。其杆组自由度可以大于 0 , 小于 0 或者等 于 0 。对于第 $k$ 个杆组, 其自由度公式可写为

$$
F_{k}=\sum_{i=1}^{P} f_{i}-\operatorname{dim}\left(G_{k}^{g z}\right)
$$

式中, $F_{k}$ 为第 $k$ 个杆组的自由度, $G_{k}^{g z}$ 为第 $k$ 个杆 组的位移参数集合, $P$ 为第 $k$ 个杆组中运动副的数 目, $f_{i}$ 为第 $i$ 个运动副的自由度数。

用 $G_{k}^{g z}=(\alpha \beta \gamma, x y z)$ 表示第 $k$ 个杆组的位移参 数, 其中 $\alpha 、 \beta 、 \gamma 、 x 、 y 、 z$ 是形式参数, 分别代表 含有绕 $x 、 y 、 z$ 轴的转动和移动。形式参数的值只 有 0 和 1 两种。 0 表示没有对应的位移， 1 表示该方 向存在位移。用 $d_{k}^{g z}$ 表示 $G_{k}^{g z}$ 的维数, 即 $d_{k}^{g z}=\operatorname{dim}\left(G_{k}^{g z}\right)$ 。

\section{2 基础杆组}

杆组结构取决于运动副的类型和相邻运动副的 轴线间的几何关系。常见运动副包括: 转动副(R), 移动副 $(\mathrm{P})$, 圆柱副 $(\mathrm{C})$, 螺旋副 $(\mathrm{H})$, 球副 $(\mathrm{S})$, 虎克 绞 $(\mathrm{U})$ 。运动副之间的轴线关系包括: 平行 $(\|)$, 同 心 $(\odot)$, 相交 $(\wedge)$, 垂直 $(\perp)$, 任意联接 $(-)$ 。

杆组是由基本杆组按照一定的几何关系联接而 成, 建立坐标系时, 要遵守最小位移参数原则, 尽 量让所有的位移参数在坐标轴上。本文列举了 8 种 基础杆组的位移参数, 如表 1 所示。

\section{3 杆组综合}

对于多环路机构, 要综合三移一转输出的机构, 杆组必须满足以下条件

$$
G_{k}^{g z} \supseteq(\alpha 00, x y z)
$$


表 1 基础杆组的位移参数

序号 分类

根据式(1), 在不同自由度下, 确定杆组位移参 数 $d_{k}^{g z}$ 和自由度数 $\sum_{i=1}^{P} f_{i}$, 再根据所得自由度和位移 参数，得到运动副之间的几何关系。通过组合基础 杆组, 综合出三移一转参数的杆组如表 2 所示。

\section{表 2 三移一转位移参数的杆组综合}

\begin{tabular}{|c|c|c|c|}
\hline $\begin{array}{c}\text { 杆组 } \\
\text { 自由度 }\end{array}$ & 位移参数 & $\begin{array}{l}\text { 运动副 } \\
\text { 自由度 }\end{array}$ & 杆组结构 \\
\hline \multirow{6}{*}{0} & & & $\mathrm{P} \perp \mathrm{R}\left\|\mathrm{C}, \mathrm{P} \perp \mathrm{P} \perp^{\perp} \mathrm{P}\right\| \mathrm{R}$, \\
\hline & $(\alpha 00, x y z)$ & 4 & $\mathrm{R}\|\mathrm{R}\| \mathrm{C}$ \\
\hline & & & $\mathrm{P}\|\mathrm{R}\| \mathrm{R}\|\mathrm{R}, \mathrm{P} \perp \mathrm{P}\| \mathrm{R} \| \mathrm{R}$ \\
\hline & $(\alpha \beta 0, x y z)$ & 5 & $\mathrm{R}\|\mathrm{R}\| \mathrm{R} \perp \mathrm{R} \| \mathrm{R}$ \\
\hline & $(\alpha \beta \gamma \gamma \gamma, 7)$ & & $\begin{array}{c}\mathrm{P}\|\mathrm{R}\| \mathrm{R} \perp \mathrm{R}\|\mathrm{R}, \mathrm{K}\| \mathrm{K} \| \mathrm{C} \perp \mathrm{K} \\
\mathrm{P} \perp \mathrm{U}-\mathrm{S}\end{array}$ \\
\hline & $(\alpha 00, x y z)$ & 3 & $\mathrm{R}\|\mathrm{C}, \quad \mathrm{P}\| \mathrm{R} \| \mathrm{R}$ \\
\hline \multirow[t]{2}{*}{-1} & $(\alpha \beta 0, x y z)$ & 4 & $\begin{array}{c}\mathrm{R}\|\mathrm{R} \perp \mathrm{R}\| \mathrm{R}, \quad \mathrm{P}\|\mathrm{R}\| \mathrm{R} \perp \mathrm{R} \\
\mathrm{R} \| \mathrm{C} \perp \mathrm{R}\end{array}$ \\
\hline & $(\alpha \beta \gamma, x y z)$ & 5 & $\mathrm{R} \perp \mathrm{R}-\mathrm{S}, \mathrm{U} \| \mathrm{U} \perp \mathrm{R}$ \\
\hline-2 & 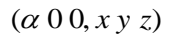 & 2 & $\mathrm{H} \| \mathrm{R}$ \\
\hline
\end{tabular}

注: $\mathrm{P} \perp \mathrm{P} \perp^{\perp} \mathrm{P} \| \mathrm{R}$ 中的 $\perp^{\perp} \mathrm{P}$ 表示该 $\mathrm{P}$ 副垂直第一个 $\mathrm{P}$ 副同时也垂 直第二个运动副 $P$ 。

\section{4 位移参数的交集运算}

为了综合三移一转位移参数机构, 本文给出了 5 种杆组位移参数的交集运算形式，如表 3 所示。

表 3 三移一转参数的组合形式

\begin{tabular}{|c|c|c|}
\hline 序号 & 组合形式 & 条件 \\
\hline 1 & $\left(\begin{array}{llllll}\alpha_{1} & 0 & 0, x_{1} & y_{1} & z_{1}\end{array}\right) \cap\left(\begin{array}{lllll}\alpha_{2} & 0 & 0, x_{2} & y_{2} & z_{2}\end{array}\right)$ & $\alpha_{1} \| \alpha_{2}$ \\
\hline 2 & 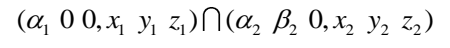 & $\alpha_{1} \| \diamond \alpha_{2} \beta_{2}$ \\
\hline 3 & $\left(\begin{array}{lllll}\alpha_{1} & 0 & 0, x_{1} & y_{1} & z_{1}\end{array}\right) \cap\left(\begin{array}{lllll}\alpha_{2} & \beta_{2} & \gamma_{2}, x_{2} & y_{2} & z_{2}\end{array}\right)$ & 无要求 \\
\hline 4 & $\left(\begin{array}{lllll}\alpha_{1} & \beta_{1} & 0, x_{1} & y_{1} & z_{1}\end{array}\right) \cap\left(\begin{array}{lllll}\alpha_{2} & 0 & 0, x_{2} & y_{2} & z_{2}\end{array}\right)$ & $\diamond \alpha_{1} \beta_{1} \| \alpha_{2}$ \\
\hline 5 & 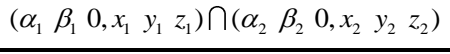 & $\diamond \alpha_{1} \beta_{1} H \diamond \alpha_{2} \beta_{2}$ \\
\hline
\end{tabular}

\section{2 输出构件自由度和位移参数分析}

对于空间多环路机构综合，其关键是找到杆组 位移参数和自由度对输出构件的关系，本节分析了 从单环路到多环路, 逐步添加杆组对输出构件的自 由度和位移参数的影响。

\section{1 单环路机构}

$L$ 个环路的并联机构是由 $L+1$ 个杆组和输出构 件所组成。其自由度公式为

$$
F_{L}=\sum_{k=1}^{L+1} F_{k}^{g z}+d_{L+1}^{M, J}
$$

式中, $d_{L+1}^{M, J}$ 表示 $L+1$ 个杆组位移参数的交集, 也为 输出构件的位移参数。

对单环路机构, 它由输出构件和两个杆组组成, 如图 1 所示。 $J$ 为参考基点, $M$ 为输出构件上的点, 输出构件位移参数的维数可以表示为

$$
d_{2}^{M, J}=\operatorname{dim}\left(\bigcap_{k=1}^{2} G_{k}^{g z}\right)
$$

式中, $d_{2}^{M, J}$ 是输出构件上基点 $M$ 的位移参数的 维数。

由式(3)可得单环路的自由度为

$$
F_{1}=F_{1}^{g z}+F_{2}^{g z}+d_{2}^{M, J}
$$

式中， $F_{1}$ 是单环路自由度。

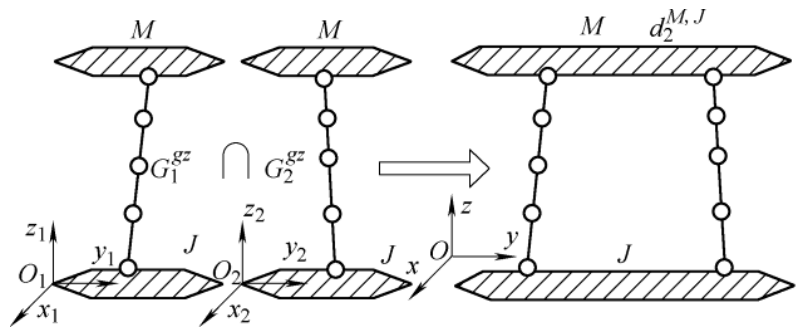

图 1 单环路机构的形成

\section{2 双环路机构}

对于双环路的机构，可以看作单环路机构和一 个杆组组成, 如图 2 所示。输出构件的参数为 


$$
d_{3}^{M, J}=\operatorname{dim}\left(G_{1}^{g z} \cap G_{2}^{g z} \cap G_{3}^{g z}\right)
$$

式中, $d_{3}^{M, J}$ 是输出构件的位移参数的维数。

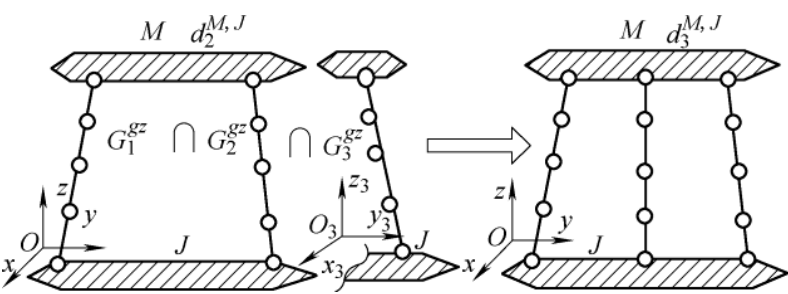

图 2 双环路机构的形成

由式(3)可得，双环路机构自由度公式为

$$
F_{2}=F_{1}^{g z}+F_{2}^{g z}+F_{3}^{g z}+d_{3}^{M, J}
$$

式中， $F_{2}$ 表示双环路的机构自由度。

如果 $F_{i}>0$, 则杆组有局部自由度, 该自由度 不影响输出构件的自由度和位移参数, 本文不考虑 这种情况。由于综合出的机构只有三移一转位移参 数, 可得

$$
d_{3}^{M, J}=4 \quad 2 \leqslant F_{2} \leqslant 4
$$

将式(8)代入式(7)可得

$$
-2 \leqslant F_{1}^{g z}+F_{2}^{g z}+F_{3}^{g z} \leqslant 0
$$

在不考虑杆组局部自由度的情况下, 第 $i$ 个杆 组的自由度需要满足以下条件

$$
F_{i}^{g z} \leqslant 0
$$

由式(9)、(10)可得, 至少一个杆组的自由度为 0 。为了避免综合出的机构重合, 设第三个杆组的自 由度为 0 , 即

$$
F_{3}^{g z}=0
$$

将式(5)、(10)代入式(6)得

$$
F_{2}=F_{1}+d_{2}^{M, J}-d_{3}^{M, J}
$$

添加杆组后输出构件位移参数 $d_{3}^{M, J}$ 与添加杆 组前输出构件位移参数 $d_{2}^{M, J}$ 必须满足以下关系

$$
d_{2}^{M, J} \leqslant d_{3}^{M, J}
$$

根据式(7)、(13)可知, 综合多环路机构关键是 确定 $d_{3}^{M, J}$ 与 $d_{4}^{M, J}$ 的的关系, 分两种情况讨论。

(1) $d_{3}^{M, J}=d_{2}^{M, J}$ 。

式(12)可简化为

$$
F_{2}=F_{1}
$$

(2) $d_{3}^{M, J}<d_{2}^{M, J}$ 。

式(12)可简化为

$$
F_{2}<F_{1}
$$

由式(15)可知, 添加第三个杆组, 机构自由度 变化。添加的第三个杆组必须对原输出构件有转动
约束。

对于三移一转位移参数的机构, 只有两个转动 约束, 最多需要两个杆组就可以提供转动约束。为 了避免重复, 第三个杆组对杆组位移参数不影响。 因此，这种情况不存在。

通过以上分析可知, 添加第三个杆组, 输出构 件自由度和位移参数不变。

\section{3 多环路机构}

对于多环路机构，可以看作在双环路机构上添 加一个杆或多个杆组。将式(3)代入式(7)得

$$
F_{L}=F_{2}+\sum_{k=4}^{L+1} F_{k}^{g z}+d_{L+1}^{M, J}-d_{3}^{M, J}
$$

根据式(8) (10)可得

$$
\sum_{k=4}^{L+1} F_{k}^{g z}=0
$$

对于三移一转位移参数的机构, 最多有两个杆 组给输出构件提供转动约束, 添加三个以上杆组后, 输出构件的位移参数不变, 也就是 $d_{3}^{M, J}=4$ 。根据 式(16)、(17), 添加自由度为 0 的杆组, 其机构自由 度不变。即

$$
F_{L}=F_{2}
$$

\section{4 空间多环路机构的综合步骤}

通过以上分析，得到了空间三移动输出机构的 综合流程图如图 3 所示。

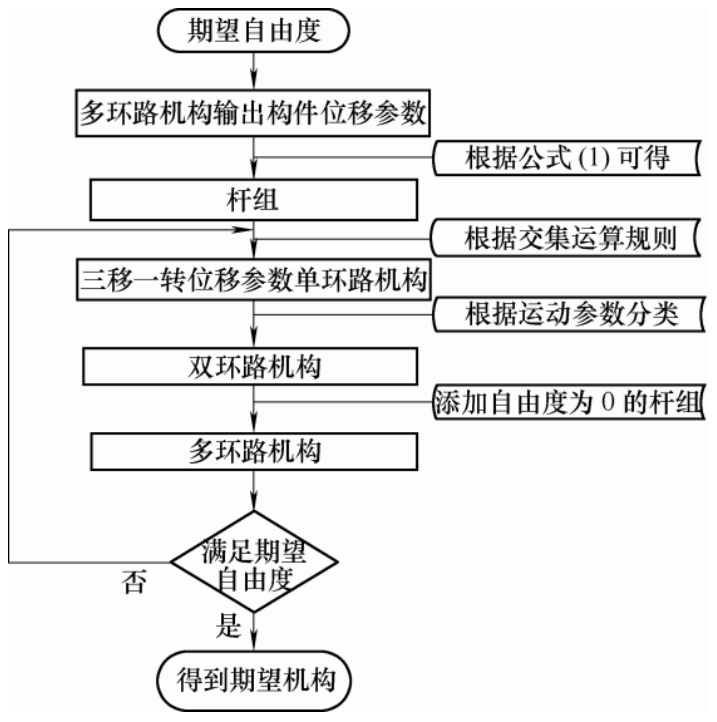

图 3 多环路综合流程图

综合多环路三移一转位移参数机构的步骤 如下。

(1) 设计杆组。根据公式(1), 确定杆组位移参 数 $d_{k}^{g z}$ 和自由度数 $\sum_{i=1}^{P} f_{i}$, 再根据表 1 得到运动副和 运动副之间的几何关系。 
（2）综合单环路机构。综合三个移动和一个转 动位移参数的机构。假设输出构件的自由度为 $2 、 3$ 和 4, 根据式(2)可得两个杆组自由度, 根据交集运 算法则, 可得到一定结构关系的两个杆组所组成的 机构。

（3）逐步在输出构件上添加一个自由度为 0 的 杆组, 得到多环路机构。

\section{3 单环路机构综合}

\section{1 综合二自由度机构}

对输出构件, 它的输出参数可能独立, 也可能 不独立。若输出的 4 个位移参数, 只有 2 个独立参 数, 则单环路的自由度为 2 , 即

$$
d_{2}^{M, J}=4, F_{1}=2
$$

将式(19)代入式(5)得

$$
F_{1}^{g z}+F_{2}^{g z}=-2
$$

式中, $F_{1}^{g z}$ 是第一个杆组的自由度, $F_{2}^{g z}$ 是第二个杆 组的自由度。

根据杆组自由度的取值范围, 每个杆组的自由 度数可分为两类。

(1) $F_{1}^{g z}=-1, \quad F_{2}^{g z}=-1$ 。
在这种情况下，假设选择 RCR 作为第一个杆 组, 该杆组有两个转动位移参数, 其位移参数为 $\left(\alpha_{1} 0 \gamma_{1}, x_{1} y_{1} z_{1}\right)$ 。为了满足式(4), 第二个杆组必然对第 一个杆组有一个转动约束, 根据表 2 的交集运算规则, 第二个杆组的位移参数有四种可能: $\left(\alpha_{2} 00, x_{2} y_{2} z_{2}\right)$, $\left(\alpha_{2} \beta_{2} 0, x_{2} y_{2} z_{2}\right),\left(\begin{array}{lll}0 & 0 & \gamma_{2}\end{array}, x_{2} y_{2} z_{2}\right),\left(0 \beta_{2} \gamma_{2}, x_{2} y_{2} z_{2}\right)$ 。 根据表 3, 假设 RCR 作为第二个杆组, 其位移 参数为 $\left(\alpha_{2} 0 \gamma_{2}, x_{2} y_{2} z_{2}\right)$ 。由于第一个杆组有绕 $x_{1}$ 和 $z_{1}$ 转动, 第二个杆组必然对第一个杆组有一个转 动约束, 根据表 2 的交集运算规则, 两杆组的几何 关系可写为 $\diamond \alpha_{1} \gamma_{1} \# \diamond \beta_{2} \gamma_{2}$ 。为了简化, 设第二个杆 组所有转动副轴线方向与 $x_{1}$ 轴线垂直, 如图 4 所示。

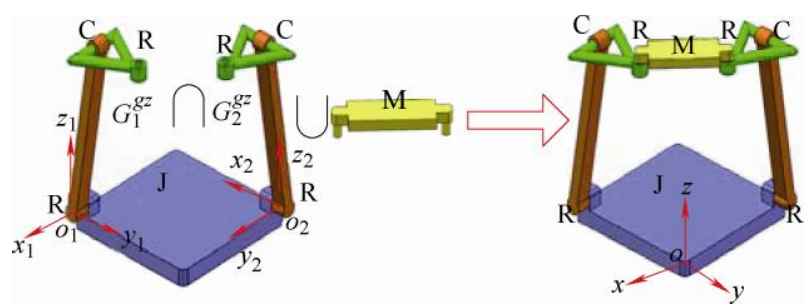

图 4 2-RCR 机构的形成

不失一般性, 选择自由度为 -1 的组杆作为第一 个杆组, 与自由度为 -1 的组杆作为第二个杆组进行 组合，进而得到一些机构如表 4 所示。

\begin{tabular}{|c|c|c|c|c|c|c|}
\hline \multirow{2}{*}{$\begin{array}{l}\text { 序 } \\
\text { 号 }\end{array}$} & \multicolumn{2}{|c|}{ 杆组 1} & \multicolumn{2}{|c|}{ 杆组 2} & \multirow{2}{*}{ 条件 } & \multirow{2}{*}{ 组合 } \\
\hline & 结构 & 位移参数 & 结构 & 位移参数 & & \\
\hline 1 & $\mathrm{RC}, \mathrm{PRR}$ & 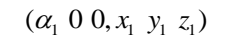 & RC, PRR & $\left(\begin{array}{lllll}\alpha_{2} & 0 & 0, x_{2} & y_{2} & z_{2}\end{array}\right)$ & $\alpha_{1} \| \alpha_{2}$ & 2-RC,RC-PRR,2-PRR \\
\hline 2 & RC,PRR & $\left(\begin{array}{llll}\alpha_{1} & 0 & 0, x_{1} & y_{1} \\
z_{1}\end{array}\right)$ & $\begin{array}{c}\text { RRRR, } \\
\text { RCR,PRRR }\end{array}$ & 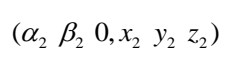 & $\alpha_{1} \| \diamond \alpha_{2} \beta_{2}$ & $\begin{array}{c}\text { RC-RRRR,RC-RCR,RC-PRRR,PRR-RR } \\
\text { RR,PRR-RCR,PRR-PRRR }\end{array}$ \\
\hline 3 & $\begin{array}{c}\text { RRRR, } \\
\text { RCR,PRRR }\end{array}$ & 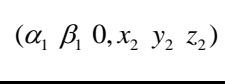 & $\begin{array}{c}\text { RRRR, } \\
\text { RCR,PRRR }\end{array}$ & 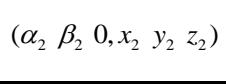 & $\diamond \alpha_{1} \beta_{1} \# \diamond \alpha_{2} \beta_{2}$ & $\begin{array}{c}\text { 2-RRRR,RRRR-RCR,RRRR-PRRR, } \\
\text { 2-RCR,RCR-PRRR,2-PRRR }\end{array}$ \\
\hline
\end{tabular}

表 $4 \quad F_{1}=-1, \quad F_{2}=-1$ 的机构

表 4 中 RC-RRRR 机构分别如图 5 所示。

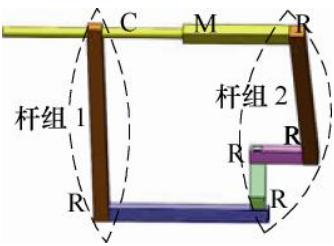

图 5 RC-RRRR 机构

(2) $F_{1}^{g z}=-2, \quad F_{2}^{g z}=0$ 。

同理, 选择一个自由度为 -2 杆组, 然后与自由 度为 0 的第二个杆组组合, 可以综合出一些机构如 表 5 所示。

\section{2 综合三自由度机构}

当输出构件有 3 个独立的位移参数, 则单环路 机构的自由度为 3 , 即

$$
d_{2}^{M, J}=4 \quad F_{1}=3
$$

将式(21)代入式(5)得

$$
F_{1}^{g z}+F_{2}^{g z}=-1
$$

为了避免综合出的机构重复, 可让 $F_{1}^{g z}=-1$, $F_{2}^{g z}=0$, 同理, 可综合出一些机构如表 6 所示, 表 6 中 RC-UUR 机构如图 6 所示。

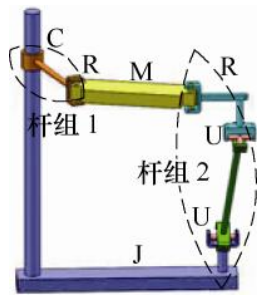

图 6 RC-UUR 机构 
表 $5 \quad F_{1}=-2, F_{2}=0$ 的机构

\begin{tabular}{|c|c|c|c|c|c|c|}
\hline \multirow{2}{*}{$\begin{array}{l}\text { 序 } \\
\text { 号 }\end{array}$} & \multicolumn{2}{|r|}{ 杆组 1} & \multicolumn{2}{|r|}{ 杆组 2} & \multirow{2}{*}{ 条件 } & \multirow{2}{*}{ 组合 } \\
\hline & 结构 & 位移参数 & 结构 & 位移参数 & & \\
\hline 1 & RH & $\left(\begin{array}{lllll}\alpha_{1} & 0 & 0, x_{1} & y_{1} & z_{1}\end{array}\right)$ & $\begin{array}{l}\text { RPC, PPPR,RRC, } \\
\text { PRRR,PPRR }\end{array}$ & $\left(\begin{array}{llllll}\alpha_{2} & 0 & 0, x_{2} & y_{2} & z_{2}\end{array}\right)$ & $\alpha_{1} \| \alpha_{2}$ & $\begin{array}{c}\text { RH-RPC,RH-PPPR,RH-RRC,RH-PR } \\
\text { RR,RH-PPRR }\end{array}$ \\
\hline 2 & $\mathrm{RH}$ & $\left(\begin{array}{lllll}\alpha_{1} & 0 & 0, x_{1} & y_{1} & z_{1}\end{array}\right)$ & $\begin{array}{c}\text { RRRRR, } \\
\text { PRRRR,RRCR }\end{array}$ & $\left(\alpha_{2} \beta_{2} \quad 0, x_{2} y_{2} z_{2}\right)$ & $\alpha_{1} \| \diamond \alpha_{2} \beta_{2}$ & RH-RRRRR, RH-PRRRR,RH-RRCR \\
\hline 3 & RH & $\left(\begin{array}{lllll}\alpha_{1} & 0 & 0, x_{1} & y_{1} & z_{1}\end{array}\right)$ & UPS & 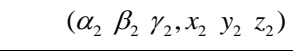 & 无要求 & RH-UPS \\
\hline \multicolumn{7}{|c|}{ 表 $6 F_{1}=-1, F_{2}=0$ 的机构 } \\
\hline \multirow{2}{*}{ 序 } & \multicolumn{2}{|r|}{ 杆组 1} & \multirow{2}{*}{\multicolumn{2}{|c|}{ 杆组 2}} & \multirow{2}{*}{ 条件 } & \multirow{2}{*}{ 组成 } \\
\hline & 结构 & 位移参数 & 结构 & & & \\
\hline 1 & RC,PRR & $\left(\begin{array}{lllll}\alpha_{1} & 0 & 0, x_{1} & y_{1} & z_{1}\end{array}\right)$ & $\begin{array}{c}\text { RPC, } \\
\text { PPPR,RRC,PRR } \\
\text { R,PPRR }\end{array}$ & $\left(\begin{array}{lllll}\alpha_{2} & 0 & 0, x_{2} & y_{2} & z_{2}\end{array}\right)$ & $\alpha_{1} \| \alpha_{2}$ & $\begin{array}{c}\text { RC-RPC,RC-PPPR,RC-RRC,RC-PRRR,RC- } \\
\text { PPRRPRR-RPC,PRR-PPPR,PRR-RRC,PRR- } \\
\text { PRRR,PRR-PPRR }\end{array}$ \\
\hline 2 & RC,PRR & $\left(\begin{array}{lllll}\alpha_{1} & 0 & 0, x_{1} & y_{1} & z_{1}\end{array}\right)$ & $\begin{array}{c}\text { RRRRR, } \\
\text { PRRRR,RRCR }\end{array}$ & $\left(\alpha_{2} \beta_{2} 0, x_{2} y_{2} z_{2}\right)$ & $\alpha_{1} \| \diamond \alpha_{2} \beta_{2}$ & $\begin{array}{c}\text { RC-RRRRR,RC-PRRRR,RC-RRCR,PRR-RR } \\
\text { RRR,PRR-PRRRR,PRR-RRCR }\end{array}$ \\
\hline 3 & $\mathrm{RC}, \mathrm{PRR}$ & $\left(\begin{array}{lllll}\alpha_{1} & 0 & 0, x_{1} & y_{1} & z_{1}\end{array}\right)$ & UPS & 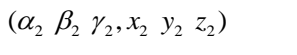 & 无要求 & RC-UPS,PRR-UPS \\
\hline 4 & $\begin{array}{l}\text { RRRR, } \\
\text { RCR,PRR } \\
\quad \mathrm{R}\end{array}$ & 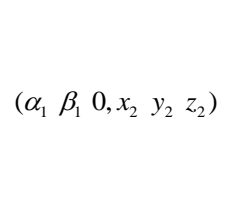 & $\begin{array}{c}\text { RPC, } \\
\text { PPPR,RRC, } \\
\text { PRRR,PPRR }\end{array}$ & 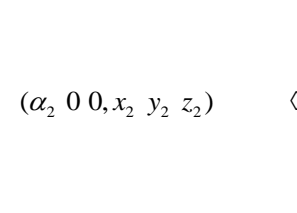 & $\diamond \alpha_{1} \beta_{1} \| \alpha_{2}$ & $\begin{array}{c}\text { RRRR-RPC,RRRR-PPPR,RRRR-RRC,RRRR } \\
\text {-PRRR,RRRR-PPRR,RCR-RPC,RCR-PPPR, } \\
\text { RCR-RRC,RCR-PRRR,RCR-PPRR,PRRR-R } \\
\text { PC,PRRR-PPPR,PRRR-RRC, } \\
\text { PRRR-PRRR,PRRR-PPRR }\end{array}$ \\
\hline 5 & $\begin{array}{l}\text { RRRR, } \\
\text { RCR,PRR } \\
\text { R }\end{array}$ & 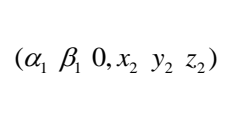 & $\begin{array}{c}\text { RRRRR, } \\
\text { PRRRR,RRCR }\end{array}$ & $\left(\alpha_{2} 0 \gamma_{2}, x_{2} y_{2} z_{2}\right)$ & $\diamond \alpha_{1} \beta_{1} H \diamond \alpha_{2}$ & $\begin{array}{l}\text { RRRR-RRRRR,RRRR-PRRRR,RRRR-RRC } \\
\text { R,RCR-RRRRR,RCR-PRRRR,RCR-RRCR,P } \\
\text { RRR-RRRRR,PRRR-PRRRR,PRRR-RRCR }\end{array}$ \\
\hline
\end{tabular}

3.3 综合 4 自由度机构

若输出构件是独立的三移一转位移参数, 则单 环路机构的自由度为 4 , 即

$$
d_{2}^{M, J}=4 \quad F_{1}=4
$$

$$
\begin{aligned}
& \text { 将式(23)代入式(5)得 } \\
& \qquad F_{1}^{g z}+F_{2}^{g z}=0
\end{aligned}
$$

根据杆组自由度的取值范围, 可以得到 $F_{1}{ }^{g z}=0$,

\begin{tabular}{|c|c|c|c|c|c|c|}
\hline \multirow{2}{*}{$\begin{array}{l}\text { 序 } \\
\text { 号 } \\
\end{array}$} & \multicolumn{2}{|c|}{ 杆组 1} & \multicolumn{2}{|r|}{ 杆组 2} & \multirow{2}{*}{ 条件 } & \multirow{2}{*}{ 组成 } \\
\hline & 结构 & 位移参数 & 结构 & 位移参数 & & \\
\hline 1 & $\begin{array}{c}\text { RPC, PPPR,RRC, } \\
\text { PRRR,PPRR }\end{array}$ & $\left(\begin{array}{lllll}\alpha_{1} & 0 & 0, x_{1} & y_{1} & z_{1}\end{array}\right)$ & $\begin{array}{c}\text { RPC, } \\
\text { PPPR,RRC, } \\
\text { PRRR,PPRR }\end{array}$ & $\left(\begin{array}{lllll}\alpha_{2} & 0 & 0, x_{2} & y_{2} & z_{2}\end{array}\right)$ & $\alpha_{1} \| \alpha_{2}$ & $\begin{array}{l}\text { 2-RPC,RPC-PPPR,RPC-RRC,RPC-PRRR,RPC-PPRR,2-P } \\
\text { PPR,PPPR-RRC,PPPR-PRRR,PPPR-PPRR2-RRC,RRC-P } \\
\text { RRR,RRC-PPRR,2-PRRR,PRRR-PPRR,2-PPRR }\end{array}$ \\
\hline 2 & $\begin{array}{c}\text { RPC, PPPR,RRC, } \\
\text { PRRR,PPRR }\end{array}$ & $\left(\begin{array}{lllll}\alpha_{1} & 0 & 0, x_{1} & y_{1} & z_{1}\end{array}\right)$ & $\begin{array}{l}\text { RRRRR, } \\
\text { PRRRR, } \\
\text { RRCR }\end{array}$ & $\left(\alpha_{2} \beta_{2} 0, x_{2} y_{2} z_{2}\right)$ & $\alpha_{1} \| \diamond \alpha_{2} \beta_{2}$ & $\begin{array}{c}\text { RPC-RRRRR,RPC-PRRRR,RPC-RRCR,PPPR-RRRRR,P } \\
\text { PPR-PRRRR,PPPR-RRCR,RRC-RRRRR,RRC-PRRRR,R } \\
\text { RC-RRCR,PRRR-RRRRR, PRRR-PRRRR,PRRR-RRCR, } \\
\text { PPRR-RRRRR,PPRR-PRRRR,PPRR-RRCR }\end{array}$ \\
\hline 3 & $\begin{array}{c}\text { RPC, PPPR,RRC, } \\
\text { PRRR,PPRR }\end{array}$ & $\left(\begin{array}{lllll}\alpha_{1} & 0 & 0, x_{1} & y_{1} & z_{1}\end{array}\right)$ & UPS & $\left(\alpha_{2} \beta_{2} \gamma_{2}, x_{2} y_{2} z_{2}\right)$ & 无要求 & RPC-UPS, PPPR-UPS,RRC-UPS, PRRR-UPS,PPRR-UPS \\
\hline
\end{tabular}
$F_{2}^{g z}=0$ 。进而得到一些机构如表 7 所示。

表 $7 \quad F_{1}=0, F_{2}=0$ 的机构

表 7 中 RRC-RRRR 机构分别如图 7 所示。

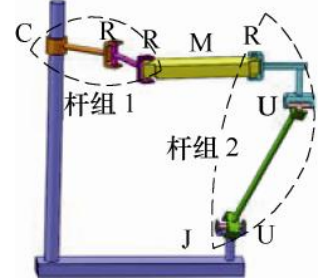

图 7 RRC-UUR 机构

\section{4 双环路机构综合}

对于三移一转位移参数的单环路机构, 添加自 由度为 0 的杆组, 其输出构件的自由度和运动参数 不变。

表 3 中的 2-RCR 机构是两自由三移一转位移参 数的单环路机构, 为了得到综合一个双环路机构, 
根据式(14), 只需要添加一个杆组。选择 $5 R$ 作为第三 个杆组, 然后与 2-RCR 机构组合, 得到 RCR-RCR-5R 机构, 如图 8 所示。

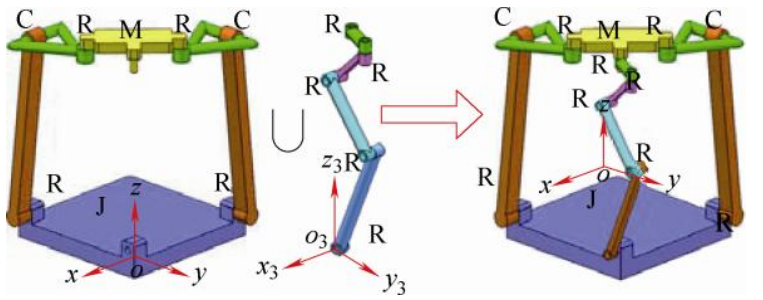

图 8 RCR-RCR-5R 机构的形成

同理, 对只有三移一转位移参数的单环路机构,
通过添加第三个自由度为 0 的杆组, 可以得到一些 双环路机构如表 8 所示。

通过组合 2-RRRR 机构和 RRRRR 杆组, 可得 到 RRRR-RRRR-RRRRR 机构如图 9 所示。

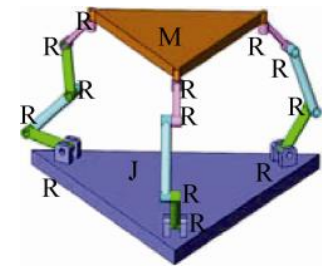

图 9 RRRR-RRRR-RRRRR 机构

表 8 双环路机构综合

\begin{tabular}{|c|c|c|c|c|}
\hline 序号 & 单环路机构 & 杆组 3 & 自由度 & 条件 \\
\hline 1 & $\begin{array}{c}\text { 2-RC,RC-PRR,2-PRR,RC-RRRR,RC-RCR,RC-PRRR,PRR-RRRR,PRR-RCR, } \\
\text { PRR-PRRR,2-RRRR,RRRR-RCR,RRRR-PRRR,2-RCR,RCR-PRRR ,2-PRRR, } \\
\text { RH-RPC,RH-PPPR,RH-RRC,RH-PRRR,RH-PPRR,RH-RRRRR,RH-PRRRR, } \\
\text { RH-RRCR,RH-UPS }\end{array}$ & $\begin{array}{l}\text { RPC,PPPR,RRC, } \\
\text { PRRR,PPRR, } \\
\text { RRRRR,PRRRR,RR } \\
\text { CR,UPS }\end{array}$ & 2 & 无要求 \\
\hline 2 & $\begin{array}{c}\text { RC-RPC,RC-PPPR,RC-RRC,RC-PRRR,RC-PPRRPRR-RPC,PRR-PPPR,PRR-RRC, } \\
\text { PRR-PRRR,PRR-PPRR,RC-RRRRR,RC-PRRRR,RC-RRCR,PRR-RRRRR,PRR-PR } \\
\text { RRR,PRR-RRCR,RC-UPS,PRR-UPS,RRRR-RPC,RRRR-PPPR, } \\
\text { RRRR-RRC,RRRR-PRRR,RRRR-PPRR,RCR-RPC,RCR-PPPR,RCR-RRC, } \\
\text { RCR-PRRR,RCR-PPRR,PRRR-RPC,PRRR-PPPR,PRRR-RRC,PRRR-PRRR, } \\
\text { PRRR-PPRR,RRRR-RRRRR,RRRR-PRRRR,RRRR-RRCR,RCR-RRRRR, } \\
\text { RCR-PRRRR,RCR-RRCR,PRRR-RRRRR,PRRR-PRRRR,PRRR-RRCR }\end{array}$ & $\begin{array}{l}\text { RPC,PPPR,RRC, } \\
\text { PRRR,PPRR, } \\
\text { RRRRR,PRRRR,RR } \\
\text { CR,UPS }\end{array}$ & 3 & 无要求 \\
\hline 3 & $\begin{array}{c}\text { 2-RPC,RPC-PPPR,RPC-RRC,RPC-PRRR,RPC-PPRR,2-PPPR,PPPR-RRC, } \\
\text { PPPR-PRRR,PPPR-PPRR2-RRC,RRC-PRRR,RRC-PPRR,2-PRRR, } \\
\text { PRRR-PPRR,2-PPRR,RPC-RRRRR,RPC-PRRRR,RPC-RRCR,PPPR-RRRRR, } \\
\text { PPPR-PRRRR,PPPR-RRCR,RRC-RRRRR,RRC-PRRRR,RRC-RRCR, } \\
\text { PRRR-RRRRR,PRRR-PRRRR,PRRR-RRCR,PPRR-RRRRR,PPRR-PRRRR, } \\
\text { PPRR-RRCR,RPC-UPS,PPPR-UPS,RRC-UPS,PRRR-UPS,PPRR-UPS }\end{array}$ & $\begin{array}{l}\text { RPC,PPPR,RRC, } \\
\text { PRRR,PPRR, } \\
\text { RRRRR,PRRRR,RR } \\
\text { CR,UPS }\end{array}$ & 4 & 无要求 \\
\hline
\end{tabular}

\section{5 多环路机构综合}

对于多环路机构, 需要在综合出的双环路机构 上添加一个或更多的自由度为 0 的杆组, 根据式 (18), 添加后机构自由度和位移参数不变。

以 RCR-RCR-5R 为例, 为例综合多环路机构, 逐个添加自由度为 0 的杆组。根据表 3 , 选择 $5 \mathrm{R}$ 作 为第四个杆组, 然后添加在 RCR-RCR-5R 机构上, 得到 RCR-RCR-5R-5R 机构, 如图 10 所示。其机构 自由度不变。根据式(18), 添加更多自由度为 0 的 杆组, 其自由度仍然不变。

由图 4 10 可以看出应用广义杆组理论综合多 环路机构是一种有效简单的方法。

\section{6 结论}

（1）基于广义杆组概念，本文提出一种针对多
环路三移一转位移输出机构综合方法。并综合一些 新机构，验证了综合方法的全面性和有效性。
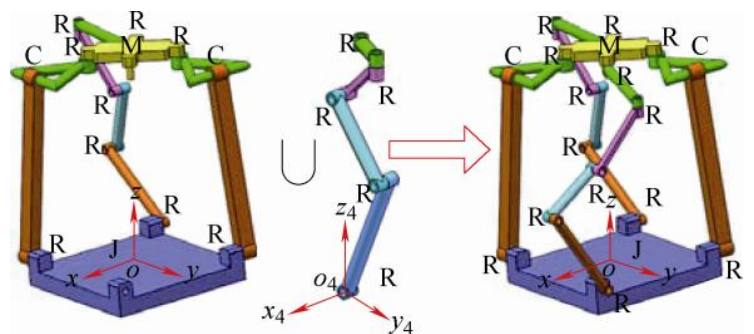

图 10 RCR-RCR-5R-5R 机构的形成

（2）该方法是以位移输出形式来综合机构，既 适用于对称机构，又适用于非对称机构，同时也适 用于独立参数和非独立参数输出机构。

（3）针对三个转动约束的机构, 本文分析了在 输出构件上添加杆组它的自由度和位移参数的影 响。进一步扩展 Assur 杆组在空间的应用。

\section{参 考 文 献}

[1] HUANG Z, LI Q C. General methodology for type 
synthesis of lower-mobility symmetrical parallel manipulators and several novel manipulators[J]. International Journal of Robotics Research, 2002, 21(2): 131-146.

[2] HUANG Z, LI Q C. Type synthesis of symmetrical lower mobility parallel mechanisms using the constraintsynthesis method[J]. Int. J. Rob. Res., 2003, 22(1): 59-82.

[3] KONG X W, GOSSELIN C M. Type synthesis of 3T1R 4-DOF parallel manipulators based on screw theory[J]. IEEE Transactions on Robotics and Automation, 2004, 20(2): 181-190.

[4] KONG X W, GOSSELIN C M. Type synthesis of 3-DOF PPR-equivalent parallel manipulators based on screw theory and the concept of virtual chain[J]. Journal of Mechanical Design, 2005, 127(6): 1113-1121.

[5] FANG Y F, TSAI L W. Structure synthesis of a class of 4-degree of freedom and 5-degree of freedom parallel manipulators with identical limb structures[J]. International Journal of Robotics Research, 2002, 21(9): 799-810.

[6] HERVE J M. The lie group of rigid body displacements, a fundamental tool for mechanism design[J]. Mechanism and Machine Theory, 1999，34: 719-730.

[7] RICO J M, Cervantes-Sanchez J J, Tadeo-Chavez A, et al. New considerations on the theory of type synthesis of fully parallel platforms[J]. Journal of Mechanical Design, 2008, 130: 112302.

[8] MENG J, LIU G, LI Z. A geometric theory for analysis and synthesis of sub-6 dof parallel manipulators[J]. IEEE Transactions on Robotics and Automation, 2007, 23: 625-649.

[9] LI Q C, HERVE J M. Parallel mechanisms with bifurcation of schoenflies motion[J]. IEEE Transactions on Robotics, 2009, 25(1): 158-164.

[10] SALGADO O, ALTUZARRA O, PETUYA V, HERNÁNDEZ A. Synthesis and design of a novel 3T1R fully-parallel manipulator[J], Journal of Mechanical Design, 2008, 130: 042305

[11] ZENG Q, FANG Y F. Structural synthesis and analysis of serial-parallel HKM with spatial multi-loop kinematic chains[J]. Mechanism and Machine Theory, 2012, 49: 198-215.

[12] EARL C F, ROONEY J. Some kinematic structures for robot manipulator designs[J]. Journal of Mechanisms, Transmissions, and Automation in Design, 1983, 105: 15-16.

[13] LU Y, WANG Y, DING L. Type synthesis of four-degree-of-freedom parallel mechanisms using valid arrays and topological graphs with digits[J]. Proceedings of the Institution of Mechanical Engineers, Part C: Journal of Mechanical Engineering Science, 2014, 228 : 3039-3053.

[14] LU Y, LU Y, DING L, YE N. Computational derivation of valid kinematic limbs of spatial 3-DOF parallel mechanisms without redundant constraint[J]. Robotica, 2012, 30: 559-569.

[15] GOGU G. Structural synthesis of fully-isotropic parallel robots with Schonflies motions via theory of linear transformations and evolutionary morphology[J]. European Journal of Mechanics- A/Solids, 2007, 26 (2): 242-269.

[16] YANG T L, LIU A X, JIN Q, et al. Position and orientation characteristic equation for topological design of robot mechanisms[J]. Journal of Mechanical Design, 2009, 131(2): 0210011-02100117.

[17] GAO F, LI W M, ZHAO X C, et al. New kinematic structures for 2-, 3-, 4-, and 5-dof parallel manipulator designs[J]. Mechanism and Machine Theory, 2002, 37(11): 1395-1411.

[18] GAO F, YANG J L, GE J D. Type synthesis of parallel mechanisms having the second class $\mathrm{G}_{\mathrm{F}}$ sets and two dimensional rotations[J]. Journal of Mechanisms and Robotics, 2010, 3(1): 011003.

[19] MANOLESCU N I. For a united point of view in the study of the structural analysis of kinematic chains and mechanisms[J]. Journal of Mechanisms, 1968, 3: 149-169.

[20] MANOLESCU N I. A method based on Baranov Trusses, and using graph theory to find the set of planar jointed kinematic chains and mechanisms[J]. Mechanism and Machine Theory, 1973, 8: 3-22.

[21] CAMPOS A, BUDDE C, HESSELBACH J. A type synthesis method for hybrid robot structures[J]. Mechanism and Machine Theory, 2008， 43: 984-995.

[22] 李树军, 戴建生. 基于 Assur 杆组元素的平面机构的拓 扑描述 $[J]$. 机械工程学报, 2011, 47(19): 8-13.

LI Shujun, DAI Jiansheng. Topological representation of planar mechanisms based on Assur group elements[J]. Journal of Mechanical Engineering, 2011, 47(19): 8-13.

[23] ZHANG Y T, MU D J. New concept and new theory of mobility calculation for multi-loop mechanisms[J]. Science China Technological Sciences, 2010, 53(6): 1598-1604.

[24] ZHANG Y T, LI Y W, WANG L Y. A new formula of mechanism mobility based on virtual constraint loop[J]. Science China Technological Sciences, 2011, 54(10): 2768-2775.

[25] ZHANG Y T, LU W J, MU D J, et al. Novel mobility formula for parallel mechanisms expressed with mobility 
of general link-group[J]. Chinese Journal of Mechanical Engineering, 2013, 26(6): 1082-1090.

[26] 牟德君, 张一同, 张兴. 机构自由度和构件自由度的关 系和本质区别 [J]. 机械工程学报, 2018, 54(5): 74-83. MU Dejun, ZHANG Yitong, ZHANG Xing. Relations between dof of mechanism and dof of links and their essential differences[J]. Journal of Mechanical Engineering, 2018, 54(5): 74-83.

[27] COMPANY O, MARQUET F, PIERROT F. A new high-speed 4-dof parallel robot synthesis and modeling issues[J]. IEEE Trans. Rob. Autom., 2003, 19(3): 411-420.

[28] NABAT V, RODRÍGUEZ MDE LA O, COMPANY O, et al. Par4 : Very high speed parallel robot for pick-and-place[C]// Proceedings of 2005 IEEE International Conference on Intelligent Robots and Systems, IROS, 2005: 553-558.

[29] KRUT S, BENOIT M, OTA H, et al. I4: A new parallel mechanism for Scara motions[C]// Proceedings 2003 IEEE International Conference on Robotics and Automation, ICRA, 2003, 1875-1880.

[30] XIE F G. A parallel robot with scara motions and its kinematic issues $[\mathrm{C}] / /$ Proceedings of the 3rd IFtoMM International Symposium on Robotics and Mechatronics, ISRM, 2013: 53-62.

[31] RICHARD P L, GOSSELIN C M, KONG X W. Kinematic analysis and prototyping of a partially decoupled 4-DOF 3T1R parallel manipulator[J]. Journal of Mechanical Design, 2007, 129 (6): 611-616.

[32] ANGELES J. The qualitative synthesis of parallel manipulators[J]. Journal of Mechanical Design, 2004, 126: 617-624.

[33] YANG T L, JIN Q, LIU A X, et al. Structure synthesis of 4-dof (3-translation and 1-rotation) parallel robot mechanisms based on the units of single-opened-chain[C]// Proceedings of the ASME 2001 Design Engineering Technical Conference and Computers and Information in Engineering Conference, 2001 : DETC2001/DAC-21152.

[34] XIE FG, LI TM, LIU X J. Type synthesis of 4-dof parallel kinematic mechanisms based on Grassmann line geometry and atlas method[J]. Chinese Journal of Mechanical Engineering, 2013, 26(6): 1073-1081.

[35] XU L M, CHEN Q H , HE L Y, LI Q C. Kinematic analysis and design of a novel 3T1R 2-(PRR) ${ }^{2} \mathrm{RH}$ hybrid manipulator[J]. Mechanism and Machine Theory, 2017, 112: $105-122$

[36] 杨廷力, 刘安心, 罗玉峰, 等. 机器人机构结构综合方 法的基本思想、特点及其发展趋势 $[\mathrm{J}]$. 机械工程学报, 2010, 46(9): 1-11.

YANG Tingli, LIU Anxin, LUO Yufeng, et al. Basic principle, main characteristics and development tendency of methods for robot mechanism structure synthesis[J]. Chinese Journal of Mechanical Engineering, 2010, 46(9): 1-11.

作者简介: 张兴, 男, 1987 年出生, 博士研究生。主要研究方向为并联 机构及控制理论。

E-mail: xing-zhang@foxmail.com

牟德君(通信作者), 女, 1967 年出生, 副教授。主要研究方向为并联机 构的设计和分析理论。

E-mail: djmu@ysu.edu.cn

张一同, 男, 1945 年出生, 教授。主要研究方向为并联机构的设计和分 析理论。

E-mail: ytzhang@ysu.edu.cn

李维奇, 男, 1993 年出生, 硕士研究生。主要研究方向为并联机构的设 计和分析理论。

E-mail: li525123112@163.com

王洪瑞, 男, 1956 年出生, 教授, 博士研究生导师。主要研究方向为并 联机构及控制理论。

E-mail: hongrui@hbu.edu.cn 